\title{
Clinical Efficacy of Adjuvant Chemotherapy in Advanced Upper Tract Urothelial Carcinoma (pT3-T4): Real-World Data from the Taiwan Upper Tract Urothelial Carcinoma Collaboration Group
}

\author{
Chung-Yu Lin ${ }^{1}$, Han-Yu Weng ${ }^{2}$, Ta-Yao Tai ${ }^{2}$, Hsi-Chin Wu ${ }^{3,4,5}$, Wen-Chi Chen ${ }^{3,6}$, Chung-Hsin Chen ${ }^{7}$, \\ Chao-Yuan Huang ${ }^{7}$, Chi-Wen Lo ${ }^{8}$, Chih-Chin Yu ${ }^{8,9}$ (D) Chung-You Tsai ${ }^{10,11}$, Wei-Che Wu 10,12, Yuan-Hong Jiang ${ }^{13}$, \\ Yu-Khun Lee ${ }^{13}(\mathbb{D})$, Thomas Y. Hsueh ${ }^{14,15}$, Allen W. Chiu ${ }^{16}$, Bing-Juin Chiang 17,18,19, Hsu-Che Huang 18,19, \\ I-Hsuan Alan Chen ${ }^{20}$, Yung-Tai Chen ${ }^{21}$, Wei-Yu Lin 22,23,24 (D), Chia-Chang Wu 25,26,27, Yao-Chou Tsai 26,28 ' \\ Hsiang-Ying Lee ${ }^{1,29}$ iD and Wei-Ming $\mathrm{Li}^{1,29,30,31, *}$
}

check for updates

Citation: Lin, C.-Y.; Weng, H.-Y.; Tai, T.-Y.; Wu, H.-C.; Chen, W.-C.; Chen, C.-H.; Huang, C.-Y.; Lo, C.-W.; Yu, C.-C.; Tsai, C.-Y.; et al. Clinical Efficacy of Adjuvant Chemotherapy in Advanced Upper Tract Urothelial Carcinoma (pT3-T4): Real-World Data from the Taiwan Upper Tract Urothelial Carcinoma Collaboration Group. J. Pers. Med. 2022, 12, 226. https://doi.org/10.3390/jpm12020226 Academic Editor: Wojciech Krajewski

Received: 20 November 2021 Accepted: 28 January 2022 Published: 6 February 2022

Publisher's Note: MDPI stays neutral with regard to jurisdictional claims in published maps and institutional affiliations.

Copyright: (C) 2022 by the authors. Licensee MDPI, Basel, Switzerland. This article is an open access article distributed under the terms and conditions of the Creative Commons Attribution (CC BY) license (https:// creativecommons.org/licenses/by/ $4.0 /)$.
1 Department of Urology, Kaohsiung Medical University Hospital, Kaohsiung 807, Taiwan; 1060489@kmuh.org.tw (C.-Y.L.); ashum1009@hotmail.com (H.-Y.L.)

2 Department of Urology, National Cheng Kung University Hospital, College of Medicine, National Cheng Kung University, Tainan 704, Taiwan; hoogagayu2@hotmail.com (H.-Y.W.); bigbite1986@gmail.com (T.-Y.T.)

3 Department of Urology, China Medical University Hospital, Taichung 404, Taiwan; wuhc@mail.cmuh.org.tw (H.-C.W.); d0283@mail.cmuh.org.tw (W.-C.C.)

4 School of Medicine, China Medical University, Taichung 404, Taiwan

5 Department of Urology, China Medical University Beigang Hospital, Yunlin 651, Taiwan

6 Graduate Institute of Integrated Medicine, College of Chinese Medicine, China Medical University, Taichung 404, Taiwan

7 Department of Urology, National Taiwan University Hospital, College of Medicine, National Taiwan University, Taipei 100, Taiwan; mufasachen@gmail.com (C.-H.C.); cyh540909@gmail.com (C.-Y.H.)

8 Division of Urology, Department of Surgery, Taipei Tzu Chi Hospital, The Buddhist Medical Foundation, New Taipei City 231, Taiwan; chiwenlo0216@gmail.com (C.-W.L.); b91401049@gmail.com (C.-C.Y.)

9 School of Medicine, Buddhist Tzu Chi University, Hualien 970, Taiwan

10 Division of Urology, Department of Surgery, Far Eastern Memorial Hospital, New Taipei City 220, Taiwan; pgtsai@gmail.com (C.-Y.T.); wuweiche@gmail.com (W.-C.W.)

11 Department of Healthcare Information and Management, Ming Chuan University, Taipei 111, Taiwan

12 Institute of Biomedical Engineering, National Taiwan University, Taipei 106, Taiwan

13 Department of Urology, Hualien Tzu Chi Hospital, Buddhist Tzu Chi Medical Foundation and Tzu Chi University, Hualien 970, Taiwan; redeemerhd@gmail.com (Y.-H.J.); leeyukhun@gmail.com (Y.-K.L.)

14 Division of Urology, Department of Surgery, Taipei City Hospital Renai Branch, Taipei 106, Taiwan; tyjhsueh@gmail.com

15 Department of Urology, School of Medicine, National Yang Ming Chiao Tung University, Taipei 112, Taiwan

16 College of Medicine, National Yang Ming Chiao Tung University, Taipei 112, Taiwan; whchiu1216@gmail.com

17 College of Medicine, Fu-Jen Catholic University, New Taipei City 242, Taiwan; bingjuinchiang@gmail.com

18 Division of Urology, Department of Surgery, Cardinal Tien Hospital, New Taipei City 231, Taiwan; huangshuche@gmail.com

19 Department of Life Science, College of Science, National Taiwan Normal University, Taipei 116, Taiwan

20 Division of Urology, Department of Surgery, Kaohsiung Veterans General Hospital, Kaohsiung 813, Taiwan; alan_aries@yahoo.com.tw

21 Department of Urology, Taiwan Adventist Hospital, Taipei 105, Taiwan; urochen831@gmail.com

22 Division of Urology, Department of Surgery, Chang Gung Memorial Hospital, Chia-Yi 613, Taiwan; lwy0912@yahoo.com

23 Chang Gung University of Science and Technology, Chia-Yi 613, Taiwan

24 Department of Medicine, Chang Gung University, Taoyuan 333, Taiwan

25 Department of Urology, Shuang Ho Hospital, Taipei Medical University, New Taipei City 235, Taiwan; charleswjj@yahoo.com.tw

26 Department of Urology, School of Medicine, College of Medicine, Taipei Medical University, Taipei 110, Taiwan; tsai1970523@yahoo.com.tw

27 TMU Research Center of Urology and Kidney (TMU-RCUK), Taipei Medical University, Taipei 110, Taiwan

28 Department of Urology, Taipei Medical University Hospital, Taipei Medical University, Taipei 110, Taiwan

29 Department of Urology, School of Medicine, College of Medicine, Kaohsiung Medical University, Kaohsiung 807, Taiwan

30 Center for Liquid Biopsy and Cohort Research, Kaohsiung Medical University, Kaohsiung 807, Taiwan 
31 Department of Urology, Ministry of Health and Welfare, Pingtung Hospital, Pingtung 900, Taiwan

* Correspondence: wmli@kmu.edu.tw

\begin{abstract}
The clinical efficacy of adjuvant chemotherapy in upper tract urothelial carcinoma (UTUC) is unclear. We aimed to assess the therapeutic outcomes of adjuvant chemotherapy in patients with advanced UTUC (pT3-T4) after radical nephroureterectomy (RNU). We retrospectively reviewed the data of 2108 patients from the Taiwan UTUC Collaboration Group between 1988 and 2018. Comprehensive clinical features, pathological characteristics, and survival outcomes were recorded. Univariate and multivariate Cox proportional hazards models were used to evaluate overall survival (OS), cancer-specific survival (CSS), and disease-free survival (DFS). Of the 533 patients with advanced UTUC included, 161 (30.2\%) received adjuvant chemotherapy. In the multivariate analysis, adjuvant chemotherapy was significantly associated with a reduced risk of overall death (hazard ratio (HR), $0.599 ; 95 \%$ confidence interval (CI), 0.419-0.857; $p=0.005)$, cancer-specific mortality (HR, 0.598; 95\% CI, 0.391-0.914; $p=0.018$ ), and cancer recurrence (HR, $0.456 ; 95 \% \mathrm{CI}, 0.310-0.673 ; p<0.001)$. The Kaplan-Meier survival analysis revealed that patients receiving adjuvant chemotherapy had significantly better five-year OS (64\% vs. 50\%, $p=0.002)$, CSS (70\% vs. $62 \%, p=0.043)$, and DFS $(60 \%$ vs. $48 \%, p=0.002)$ rates compared to those who did not receive adjuvant chemotherapy. In conclusion, adjuvant chemotherapy after RNU had significant therapeutic benefits on OS, CSS, and DFS in advanced UTUC.
\end{abstract}

Keywords: upper tract urothelial carcinoma; chemotherapy; prognosis

\title{
1. Introduction
}

Upper tract urothelial carcinoma (UTUC) is a relatively rare tumor that accounts for $10 \%$ of all renal tumors and $5 \%$ of urothelial malignancies overall, with an estimated annual incidence of almost two cases per 100,000 inhabitants in Western countries [1,2]. However, the epidemiology and clinical presentation of UTUCs in Taiwan are quite different from those in Western populations [3-5]. In Taiwan, the incidence rate of UTUC is much higher than that worldwide and in Western populations, and the prevalence of UTUC is as high as $30 \%$ of all urothelial carcinomas (UCs) [3-5]. Several risk factors associated with UCs, such as exposure to arsenic, toxic chemicals, aristolochic acid, and cigarette smoking, have been reported in previous studies [6,7]. Radical nephroureterectomy (RNU) with bladder cuff excision is regarded as the standard therapy for local UTUC, but monotherapy with surgical intervention is considered to be associated with relatively high recurrence rates and low survival rates in the advanced pathological stage $[2,4,5,8]$. The five-year overall survival rate is less than $50 \%$ in patients with T2-3 and less than $10 \%$ in patients with T4 [2,9].

In the current study, UC is chemosensitive cancer, and the effectiveness of chemotherapy in treating urinary bladder UC has been well established $[2,10,11]$. Thus, postoperative adjuvant chemotherapy has been suggested to patients with advanced-stage UC, such as those with $\geq$ pathological T3 or node-positive disease $[2,10,11]$. However, there is less strong evidence for adjuvant chemotherapy followed by RNU in patients with UTUC. Although the current guidelines suggest that cisplatin-based perioperative chemotherapy can provide a survival benefit for patients with advanced stage, the current evidence and practice are still mainly dependent on data from bladder cancer due to the rarity of UTUC worldwide $[2,11]$.

Therefore, this study aimed to investigate the current role of postoperative adjuvant chemotherapy in patients with advanced UTUC (pathological T3-T4) in a large multicenter cohort from Taiwan and to set a benchmark for comparison of future novel trials. 


\section{Materials and Methods}

\subsection{Patient Population}

Taiwan UTUC Collaboration Group conducted this nationwide study. We included 14 participating Taiwan hospitals (Kaohsiung Medical University Hospital, Taipei Tzu Chi Hospital, Hualien Tzu Chi Hospital, Chang Gung Memorial Hospital, Chiayi, Kaohsiung Veterans General Hospital, China Medical University Hospital, Taipei City Hospital, Taiwan Adventist Hospital, National Taiwan University Hospital, Taipei Medical University Shuang Ho Hospital, Cardinal Tien Hospital, National Cheng Kung University Hospital, Taipei Medical University Hospital, and Far Eastern Memorial Hospital). This study was approved by the institutional review board (KMUHIRB-E(I)-20190107). We retrospectively reviewed the data of 2108 patients diagnosed with UTUC between 1988 and 2018. Among the 2108 patients, 1524 were excluded from this study, 293 did not undergo RNU, 1201 had early-stage UTUC (pathological Ta-T2), seven were not UC, 16 were treated with neoadjuvant chemotherapy, 41 were with positive surgical margin, and 19 were followed up $<1$ month. The remaining 584 patients with advanced disease (pathological T3-T4) who underwent RNU were included for further analysis.

The following characteristics were included: sex, age, body mass index (BMI), Eastern Cooperative Oncology Group (ECOG) performance status, American Society of Anesthesiologists (ASA) physical status classification system, smoking, tumor size, tumor number, tumor location, tumor grade, tumor stage, concurrent carcinoma in situ (CIS), lymphovascular invasion (LVI), histological variants, lymph node status, surgical margin, and adjuvant chemotherapy. Tumor staging was defined according to the 2010 American Joint Committee on Cancer TNM staging system, and tumor grade was determined based on the $2004 \mathrm{WHO}$ classification. Adjuvant chemotherapy was defined as chemotherapy administered within three months after RNU. Most patients received platinum-based chemotherapy, including methotrexate, vinblastine, doxorubicin, cisplatin/carboplatin (MVAC), or gemcitabine plus cisplatin/carboplatin. After RNU, patients received regular follow-up programs, including physical, laboratory, and radiological examinations, according to standard guidelines. UTUC recurrence was defined as local recurrence at the tumor bed, regional lymph node metastasis, or distant organ metastasis. The cause of death was determined according to death certificates.

\subsection{Statistical Analysis}

To compare the clinicopathological characteristics of the two groups (with vs. without adjuvant chemotherapy), a Student's $t$-test and Pearson's chi-square test were used for continuous and categorical variables, respectively. We used the Kaplan-Meier survival analysis to estimate the clinical outcomes, including overall survival (OS), cancer-specific survival (CSS), and disease-free survival (DFS). Univariate and multivariate Cox proportional hazard regression models with Enter method were used to identify independent prognostic factors of clinical outcomes (OS, CSS, and DFS). We used the landmark method to minimize immortal time bias. Patients with survival $>1$ month were only included to account for immortal time bias in patients who were not able to receive adjuvant chemotherapy and to control the acute surgical complications.

All statistical analyses were conducted using SPSS version 26 (IBM, Armonk, NY, USA), and $p$-values of $<0.05$ were considered statistically significant.

\section{Results}

\subsection{Patient Characteristics}

This study observed 533 patients diagnosed with advanced UTUC (pT3-T4) after standard treatment with RUN. A total of 247 males and 286 females participated in the study. The adjuvant chemotherapy group consisted of 161 (30.2\%) patients with UTUC who received chemotherapy followed by RNU. The non-adjuvant chemotherapy group comprised $372(69.8 \%)$ patients treated with initial surgical management that did not undergo adjuvant chemotherapy. The clinical characteristics of the patients are summarized 
in Table 1. The mean age of the study population was 68.6 years. The mean length of follow-up was 54.1 months. In all, 472 patients $(88.6 \%)$ were diagnosed with pT3 disease, and 61 (11.4\%) were diagnosed with pT4 disease. Regarding kidney function, the mean eGFR was $50.4 \mathrm{~mL} / \mathrm{min}$ per $1.73 \mathrm{~m}^{2}$, and $35 \%$ patients had chronic kidney disease (eGFR $<60 \mathrm{~mL} / \mathrm{min}$ ). After RNU, we found a mean difference between preoperative and postoperative eGFR of $10.1 \mathrm{~mL} / \mathrm{min}$ per $1.73 \mathrm{~m}^{2}(p<0.001)$. The median follow-up period was 46.8 months in the adjuvant chemotherapy group and significantly longer than in the non-adjuvant chemotherapy group at 29 months $(p=0.001)$. The patients receiving adjuvant chemotherapy were younger $(64.3 \pm 9.9$ vs. $70.5 \pm 11.2$ years, $p<0.001)$ and had better ASA physical status $(p=0.009)$ and lymph node metastasis $(13.7 \%$ vs. $5.4 \%, p=0.011)$ than those without chemotherapy administration. No significant differences were found in terms of sex, BMI, ECOG performance status, and tumor presentation characteristics, including tumor location, tumor size, histological variants of the tumor, tumor grade, presence of CIS, LVI, and pathological stage.

Table 1. Patients' demographic and clinicopathological characteristics.

\begin{tabular}{|c|c|c|c|c|c|}
\hline \multirow[t]{2}{*}{ Variables } & \multicolumn{2}{|c|}{$\begin{array}{c}\text { Adjuvant } \\
\text { Chemotherapy } \\
(n=161)\end{array}$} & \multicolumn{2}{|c|}{$\begin{array}{l}\text { No Adjuvant } \\
\text { Chemotherapy } \\
\quad(n=372)\end{array}$} & \multirow[t]{2}{*}{$p$-Value } \\
\hline & $n$ & $(\%)$ & $n$ & $(\%)$ & \\
\hline \multicolumn{6}{|l|}{ Sex } \\
\hline Male & 80 & $(49.7)$ & 167 & $(44.9)$ & 0.308 \\
\hline Female & 81 & (50.3) & 205 & (55.1) & \\
\hline \multicolumn{6}{|l|}{ Age (years) } \\
\hline Mean $\pm S D$ & \multicolumn{2}{|c|}{$64.3 \pm 9.9$} & \multicolumn{2}{|c|}{$70.5 \pm 11.2$} & $<0.001$ * \\
\hline Median & \multicolumn{2}{|c|}{65.9} & \multicolumn{2}{|c|}{726} & \\
\hline \multicolumn{6}{|l|}{$\mathrm{BMI}\left(\mathrm{kg} / \mathrm{m}^{2}\right)$} \\
\hline Mean $\pm S D$ & \multicolumn{2}{|c|}{$24.5 \pm 4.0$} & \multicolumn{2}{|c|}{$23.9 \pm 3.8$} & 0.386 \\
\hline Median & \multicolumn{2}{|c|}{24.1} & \multicolumn{2}{|c|}{23.8} & \\
\hline \multicolumn{6}{|l|}{ Follow-up (months) } \\
\hline Mean $\pm S E$ & \multicolumn{2}{|c|}{$60.1 \pm 7.6$} & \multicolumn{2}{|c|}{$51.2 \pm 7.8$} & $<0.001$ * \\
\hline Median & \multicolumn{2}{|c|}{46.8} & \multicolumn{2}{|c|}{29.0} & \\
\hline \multicolumn{6}{|l|}{ ASA } \\
\hline 1 & 2 & $(2.0)$ & 4 & $(2.4)$ & $0.009 *$ \\
\hline 2 & 51 & $(50.0)$ & 50 & $(29.8)$ & \\
\hline 3 & 49 & $(48.0)$ & 113 & $(67.3)$ & \\
\hline 4 & 0 & $(0.0)$ & 1 & $(0.6)$ & \\
\hline \multicolumn{6}{|l|}{ ECOG } \\
\hline 0 & 58 & $(46.8)$ & 98 & $(41.0)$ & 0.293 \\
\hline 1 & 55 & $(44.4)$ & 102 & $(42.7)$ & \\
\hline 2 & 10 & (8.1) & 31 & (13.0) & \\
\hline 3 & 1 & $(0.8)$ & 4 & (1.7) & \\
\hline 4 & 0 & $(0.0)$ & 4 & $(1.7)$ & \\
\hline \multicolumn{6}{|l|}{ Smoking } \\
\hline No & 85 & $(72.0)$ & 182 & (71.7) & 0.940 \\
\hline Yes & 33 & $(28.0)$ & 72 & $(28.3)$ & \\
\hline \multicolumn{6}{|l|}{ Tumor location } \\
\hline Renal pelvis & 80 & (49.7) & 202 & (54.3) & 0.317 \\
\hline Ureter & 50 & (31.1) & 92 & $(24.7)$ & \\
\hline Renal pelvis + ureter & 31 & (19.3) & 78 & $(21.0)$ & \\
\hline
\end{tabular}


Table 1. Cont.

\begin{tabular}{|c|c|c|c|c|c|}
\hline \multirow[t]{2}{*}{ Variables } & \multicolumn{2}{|c|}{$\begin{array}{c}\text { Adjuvant } \\
\text { Chemotherapy } \\
(n=161)\end{array}$} & \multicolumn{2}{|c|}{$\begin{array}{l}\text { No Adjuvant } \\
\text { Chemotherapy } \\
(n=372)\end{array}$} & \multirow[t]{2}{*}{$p$-Value } \\
\hline & $n$ & $(\%)$ & $n$ & $(\%)$ & \\
\hline \multicolumn{6}{|l|}{ Tumor size } \\
\hline$<1 \mathrm{~cm}$ & 3 & (2.3) & 10 & $(3.2)$ & 0.760 \\
\hline$\geq 1$ and $<2 \mathrm{~cm}$ & 22 & $(16.5)$ & 39 & $(12.7)$ & \\
\hline$\geq 2$ and $<3 \mathrm{~cm}$ & 26 & (19.5) & 56 & $(18.2)$ & \\
\hline$\geq 3 \mathrm{~cm}$ & 82 & $(61.7)$ & 203 & $(65.9)$ & \\
\hline \multicolumn{6}{|c|}{ Histological variant } \\
\hline No & 135 & $(83.9)$ & 322 & $(86.6)$ & 0.412 \\
\hline Yes & 26 & $(16.1)$ & 50 & $(13.4)$ & \\
\hline \multicolumn{6}{|l|}{ Tumor grade } \\
\hline Low grade & 12 & $(7.5)$ & 15 & $(4.1)$ & 0.098 \\
\hline High grade & 148 & $(92.5)$ & 355 & $(95.9)$ & \\
\hline \multicolumn{6}{|l|}{ Multifocality } \\
\hline No & 102 & $(63.4)$ & 234 & $(62.9)$ & 0.921 \\
\hline Yes & 59 & $(36.6)$ & 138 & $(37.1)$ & \\
\hline \multicolumn{6}{|l|}{ Concurrent CIS } \\
\hline No & 141 & $(87.6)$ & 325 & $(87.4)$ & 0.946 \\
\hline Yes & 20 & $(12.4)$ & 47 & $(12.6)$ & \\
\hline \multicolumn{6}{|c|}{ Lymphovascular invasion } \\
\hline No & 99 & $(61.5)$ & 245 & $(65.9)$ & 0.333 \\
\hline Yes & 62 & $(38.5)$ & 127 & $(34.1)$ & \\
\hline \multicolumn{6}{|c|}{ Pathological stage } \\
\hline Stage III & 128 & $(79.5)$ & 308 & $(83.2)$ & 0.301 \\
\hline Stage IV & 33 & $(83.2)$ & 62 & $(16.7)$ & \\
\hline \multicolumn{6}{|c|}{ Pathological T stage } \\
\hline pT3 & 146 & $(90.7)$ & 326 & $(87.6)$ & 0.310 \\
\hline pT4 & 15 & $(9.3)$ & 46 & $(12.4)$ & \\
\hline \multicolumn{6}{|c|}{ Pathological N stage } \\
\hline pN0 & 38 & $(23.6)$ & 74 & $(19.9)$ & 0.011 * \\
\hline pN1 & 10 & $(6.2)$ & 10 & $(2.7)$ & \\
\hline $\mathrm{pN} 2$ & 12 & (7.5) & 10 & $(2.7)$ & \\
\hline $\mathrm{pNx}$ & 98 & $(60.9)$ & 272 & $(73.1)$ & \\
\hline
\end{tabular}

\subsection{Clinical Outcomes}

Univariate analyses for OS, CSS, and DFS rates are summarized in Table 2. We found that adjuvant chemotherapy, tumor size, tumor grade, multiplicity of the tumor, and pathological stage, including $\mathrm{T}$ and $\mathrm{N}$ stage, were significantly associated with OS, CSS, and DFS. Age and lymphovascular invasion were significant prognostic factors for OS and CSS but not for DFS. Smoking history was significantly correlated with CSS but not DFS and OS. Different chemotherapy regimen was not a significant predictor for OS, CSS, and DFS.

In multivariable analysis, adjuvant chemotherapy was demonstrated to be a significant independent prognostic factor of OS (hazard ratio (HR), $0.599 ; 95 \%$ confidence interval (CI) 0.419-0.857; $p=0.005)$, CSS (HR, 0.598; 95\% CI, 0.391-0.914; $p=0.018)$, and DFS (HR, 0.456; 95\% CI, 0.310-0.673; $p<0.001$ ) in pT3-T4 UTUC patients after RUN (Table 3). We also found that high tumor grade, high pathological tumor stage, and lymph node metastasis were significantly independently associated with worse OS, CSS, and DFS. Age was a significant prognostic factor for OS but not for CSS and DFS.

The Kaplan-Meier survival analysis (Figure 1) revealed that patients with advanced UTUC who underwent adjuvant chemotherapy had significantly better five-year OS (64\% vs. $50 \%, p=0.002)$, CSS ( $70 \%$ vs. $62 \%, p=0.043)$, and DFS ( $60 \%$ vs. $48 \%, p=0.002)$ rates than 
those who did not receive adjuvant chemotherapy. In competing risk analysis, adjuvant chemotherapy improved CSS (sHR, $0.735 ; 95 \%$ CI, $0.400-1.070 ; p=0.073$ ) and DFS (sHR, $0.657 ; 95 \%$ CI, 0.367-0.947; $p=0.004$ ) (Table S1). Overall, the observed treatment effect on OS, CSS, and DFS was consistent across subgroups (Figure 2).

Table 2. Univariate analyses for overall, cancer-specific, and disease-free survivals in advanced UTUC patients.

\begin{tabular}{|c|c|c|c|c|c|c|c|c|c|}
\hline \multirow[b]{2}{*}{ Variables } & \multicolumn{3}{|c|}{ Overall Survival } & \multicolumn{3}{|c|}{ Cancer-Specific Survival } & \multicolumn{3}{|c|}{ Disease-Free Survival } \\
\hline & HR & $95 \%$ CI & $p$-Value & HR & $95 \%$ CI & $p$-Value & HR & $95 \%$ CI & $p$-Value \\
\hline \multicolumn{10}{|l|}{$\begin{array}{c}\text { Adjuvant } \\
\text { chemotherapy }\end{array}$} \\
\hline No & 1 & & & 1 & & & 1 & & \\
\hline Yes & 0.636 & $0.478-0.847$ & $0.002 *$ & 0.700 & $0.495-0.990$ & $0.044^{*}$ & 0.624 & $0.459-0.850$ & $0.003 *$ \\
\hline \multicolumn{10}{|l|}{$\begin{array}{l}\text { Chemotherapy } \\
\text { regimens }\end{array}$} \\
\hline $\begin{array}{l}\text { Gemcitabine and } \\
\text { cisplatin }\end{array}$ & 1 & & & & 1 & & & & 1 \\
\hline MVAC & 1.014 & $0.598-1.718$ & 0.960 & 0.847 & $0.475-1.511$ & 0.574 & 0.794 & $0.480-1.316$ & 0.371 \\
\hline Carboplatin-based & 0.529 & $0.127-2.204$ & 0.382 & 0.314 & $0.043-2.296$ & 0.254 & 0.482 & $0.116-1.998$ & 0.315 \\
\hline Others & 1.215 & $0.774-1.905$ & 0.397 & 1.048 & $0.640-1.714$ & 0.853 & 1.057 & $0.689-1.624$ & 0.799 \\
\hline \multicolumn{10}{|l|}{ Sex } \\
\hline Male & 1 & & & 1 & & & 1 & & \\
\hline Female & 1.011 & $0.788-1.296$ & 0.932 & 1.110 & $0.814-1.515$ & 0.509 & 1.119 & $0.851-1.472$ & 0.421 \\
\hline \multicolumn{10}{|l|}{ Age } \\
\hline$<70$ & 1 & & & 1 & & & 1 & & \\
\hline$\geq 70$ & 1.750 & $1.360-2.253$ & $<0.001$ * & 1.407 & $1.031-1.921$ & 0.031 * & 1.280 & $0.971-1.686$ & 0.080 \\
\hline \multicolumn{10}{|l|}{ BMI } \\
\hline$<24$ & 1 & & & 1 & & & 1 & & \\
\hline$\geq 24$ & 0.896 & $0.647-1.241$ & 0.509 & 0.989 & $0.678-1.444$ & 0.956 & 0.990 & $0.698-1.402$ & 0.953 \\
\hline \multicolumn{10}{|l|}{ ASA } \\
\hline 1 & 1 & & & 1 & & & 1 & & \\
\hline$>1$ & 2.527 & $0.352-18.131$ & 0.357 & 1.833 & $0.254-13.201$ & 0.548 & 1.202 & $0.296-4.885$ & 0.797 \\
\hline \multicolumn{10}{|l|}{ ECOG } \\
\hline 0 & 1 & & & 1 & & & 1 & & \\
\hline$\geq 1$ & 1.115 & $0.805-1.544$ & 0.513 & 0.978 & $0.672-1.423$ & 0.906 & 1.028 & 0.729-1.449 & 0.876 \\
\hline \multicolumn{10}{|l|}{ Smoking } \\
\hline No & 1 & & & 1 & & & 1 & & \\
\hline Yes & 1.181 & $0.849-1.643$ & 0.323 & 1.482 & $1.005-2.186$ & 0.047 * & 1.368 & $0.968-1.934$ & 0.076 \\
\hline \multicolumn{10}{|l|}{ Hospitals } \\
\hline Medical centers & 1 & & & 1 & & & 1 & & \\
\hline Regional hospitals & 1.111 & $0.880-1.403$ & 0.376 & 1.166 & $0.882-1.541$ & 0.280 & 1.105 & $0.855-1.429$ & 0.446 \\
\hline \multicolumn{10}{|l|}{ Tumor location } \\
\hline Renal pelvis & 1 & & & 1 & & & 1 & & \\
\hline Ureter & 1.134 & $0.848-1.517$ & 0.395 & 1.358 & $0.952-1.937$ & 0.091 & 1.349 & $0.979-1.858$ & 0.067 \\
\hline Renal pelvis + ureter & 1.200 & $0.869-1.657$ & 0.267 & 1.214 & $0.808-1.824$ & 0.351 & 1.289 & $0.910-1.826$ & 0.153 \\
\hline \multicolumn{10}{|l|}{ Tumor size } \\
\hline$<3 \mathrm{~cm}$ & 1 & & & 1 & & & 1 & & \\
\hline$\geq 3 \mathrm{~cm}$ & 1.478 & $1.082-2.018$ & $0.014^{*}$ & 1.898 & $1.281-2.810$ & $0.001 *$ & 1.607 & $1.154-2.240$ & $0.005^{*}$ \\
\hline \multicolumn{10}{|l|}{ Histological variants } \\
\hline No & 1 & & & 1 & & & 1 & & \\
\hline Yes & 1.185 & $0.828-1.694$ & 0.353 & 1.355 & $0.890-2.063$ & 0.156 & 1.105 & $0.754-1.620$ & 0.609 \\
\hline \multicolumn{10}{|l|}{ Tumor grade } \\
\hline Low grade & 1 & & & 1 & & & 1 & & \\
\hline High grade & 2.854 & $1.269-6.421$ & $0.011 *$ & 5.804 & $1.438-23.414$ & $0.013 *$ & 4.212 & $1.565-11.339$ & 0.004 * \\
\hline
\end{tabular}


Table 2. Cont.

\begin{tabular}{|c|c|c|c|c|c|c|c|c|c|}
\hline \multirow[b]{2}{*}{ Variables } & \multicolumn{3}{|c|}{ Overall Survival } & \multicolumn{3}{|c|}{ Cancer-Specific Survival } & \multicolumn{3}{|c|}{ Disease-Free Survival } \\
\hline & HR & $95 \%$ CI & $p$-Value & HR & $95 \%$ CI & $p$-Value & HR & $95 \%$ CI & $p$-Value \\
\hline \multicolumn{10}{|l|}{ Multifocality } \\
\hline No & 1 & & & 1 & & & 1 & & \\
\hline Yes & 1.367 & $1.062-1.758$ & $0.015 *$ & 1.407 & $1.029-1.924$ & $0.033 *$ & 1.390 & $1.053-1.836$ & $0.020 *$ \\
\hline \multicolumn{10}{|c|}{ Concurrent CIS } \\
\hline No & 1 & & & 1 & & & 1 & & \\
\hline Yes & 1.122 & $0.751-1.676$ & 0.575 & 1.125 & $0.696-1.817$ & 0.632 & 0.959 & $0.626-1.469$ & 0.847 \\
\hline \multicolumn{10}{|c|}{$\begin{array}{l}\text { Lymphovascular } \\
\text { invasion }\end{array}$} \\
\hline No & 1 & & & 1 & & & 1 & & \\
\hline Yes & 1.476 & $1.139-1.914$ & $0.003 *$ & 1.672 & $1.223-2.285$ & $0.001 *$ & 1.305 & $0.988-1.725$ & 0.061 \\
\hline \multicolumn{10}{|c|}{ Pathological T stage } \\
\hline pT3 & 1 & & & 1 & & & 1 & & \\
\hline pT4 & 2.723 & $1.980-3.746$ & $<0.001 *$ & 3.120 & $2.124-4.583$ & $<0.001 *$ & 2.294 & $1.567-3.357$ & $<0.001$ * \\
\hline \multicolumn{10}{|c|}{ Pathological N stage } \\
\hline $\mathrm{pN} 0+\mathrm{pNx}$ & 1 & & & 1 & & & 1 & & \\
\hline $\mathrm{pN} 1+\mathrm{pN} 2$ & 0.474 & $0.313-0.718$ & $<0.001 *$ & 0.389 & $0.246-0.617$ & $<0.001$ & 0.410 & $0.269-0.625$ & $<0.001 *$ \\
\hline
\end{tabular}

Table 3. Multivariate analyses for overall, cancer-specific, and disease-free survivals in advanced UTUC patients.

\begin{tabular}{|c|c|c|c|c|c|c|c|c|c|}
\hline \multirow[b]{2}{*}{ Variables } & \multicolumn{3}{|c|}{ Overall Survival } & \multicolumn{3}{|c|}{ Cancer-Specific Survival } & \multicolumn{3}{|c|}{ Disease-Free Survival } \\
\hline & HR & $95 \% \mathrm{CI}$ & $p$-Value & HR & $95 \%$ CI & $p$-Value & HR & $95 \% \mathrm{CI}$ & $p$-Value \\
\hline \multicolumn{10}{|l|}{$\begin{array}{c}\text { Adjuvant } \\
\text { chemotherapy }\end{array}$} \\
\hline No & 1 & & & 1 & & & 1 & & \\
\hline Yes & 0.599 & $0.419-0.857$ & $0.005^{*}$ & 0.598 & $0.391-0.914$ & $0.018 *$ & 0.456 & $0.310-0.673$ & $<0.001 *$ \\
\hline \multicolumn{10}{|l|}{ Age } \\
\hline$<70$ & 1 & & & 1 & & & 1 & & \\
\hline$\geq 70$ & 1.727 & $1.272-2.346$ & $<0.001 *$ & 1.321 & $0.917-1.902$ & 0.134 & 1.176 & $0.856-1.616$ & 0.317 \\
\hline \multicolumn{10}{|l|}{ Tumor size } \\
\hline$<3 \mathrm{~cm}$ & 1 & & & 1 & & & 1 & & \\
\hline$\geq 3 \mathrm{~cm}$ & 1.188 & $0.859-1.643$ & 0.299 & 1.473 & $0.974-2.226$ & 0.066 & 1.377 & 0.978-1.939 & 0.067 \\
\hline \multicolumn{10}{|l|}{ Tumor grade } \\
\hline Low grade & 1 & & & 1 & & & 1 & & \\
\hline High grade & 2.526 & $1.033-6.181$ & $0.042 *$ & 8.184 & $1.139-58.824$ & $0.037^{*}$ & 3.772 & $1.197-11.885$ & $0.023 *$ \\
\hline \multicolumn{10}{|l|}{ Multifocality } \\
\hline No & 1 & & & 1 & & & 1 & & \\
\hline Yes & 1.274 & $0.951-1.707$ & 0.104 & 1.375 & $0.966-1.957$ & 0.077 & 1.415 & $1.043-1.920$ & $0.026^{*}$ \\
\hline \multicolumn{10}{|c|}{$\begin{array}{l}\text { Lymphovascular } \\
\text { invasion }\end{array}$} \\
\hline No & 1 & & & 1 & & & 1 & & \\
\hline Yes & 1.258 & $0.935-1.693$ & 0.129 & 1.395 & $0.970-2.006$ & 0.073 & 1.193 & $0.870-1.635$ & 0.274 \\
\hline \multicolumn{10}{|c|}{ Pathological T stage } \\
\hline pT3 & 1 & & & 1 & & & 1 & & \\
\hline pT4 & 2.390 & $1.588-3.596$ & $<0.001 *$ & 2.234 & $1.377-3.627$ & $0.001 *$ & 1.654 & $1.033-2.650$ & $0.036^{*}$ \\
\hline \multicolumn{10}{|c|}{ Pathological N stage } \\
\hline $\mathrm{pNO}+\mathrm{pNx}$ & 1 & & & 1 & & & 1 & & \\
\hline $\mathrm{pN} 1+\mathrm{pN} 2$ & 2.234 & $1.397-3.572$ & $0.001 *$ & 2.323 & $1.361-3.963$ & $0.002 *$ & 2.888 & $1.755-4.752$ & $<0.001 *$ \\
\hline
\end{tabular}


A

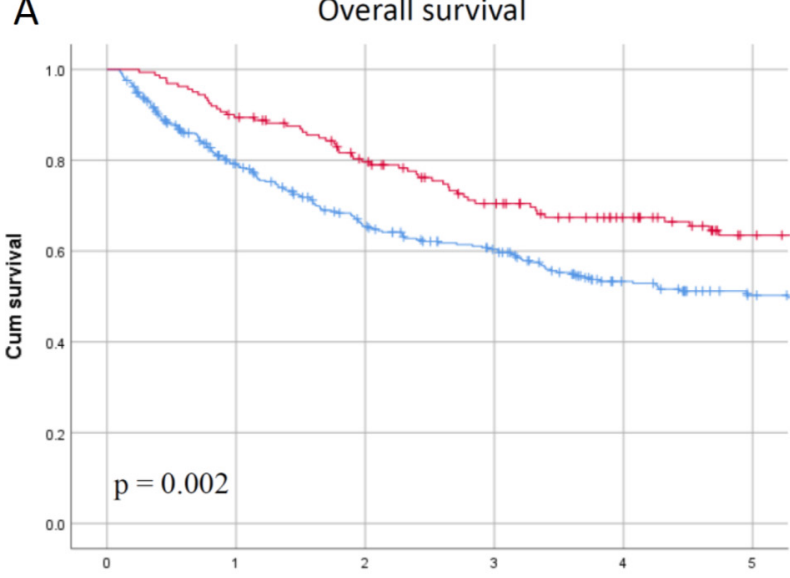

Time (year)

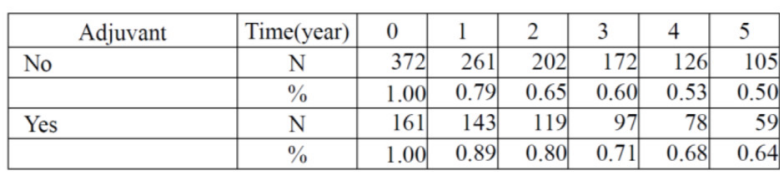

B

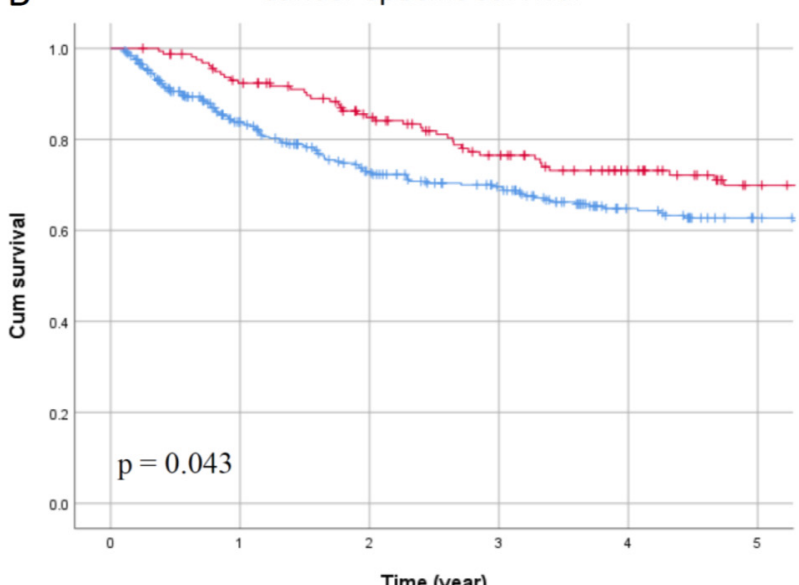

Time (year)

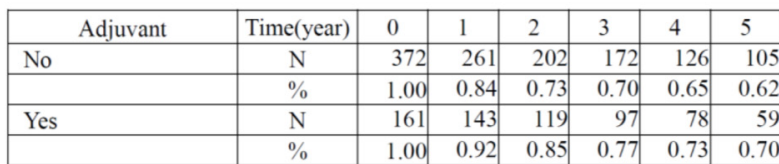

C
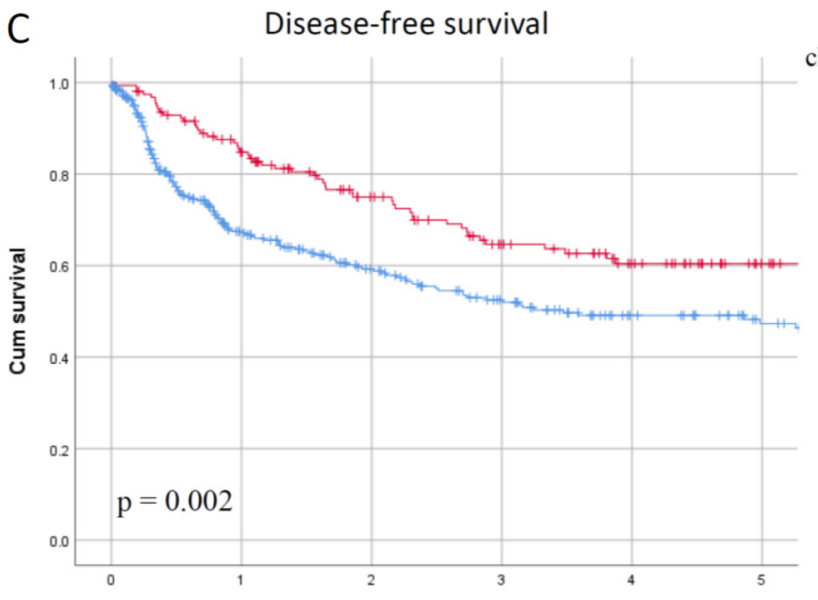

Time (year)

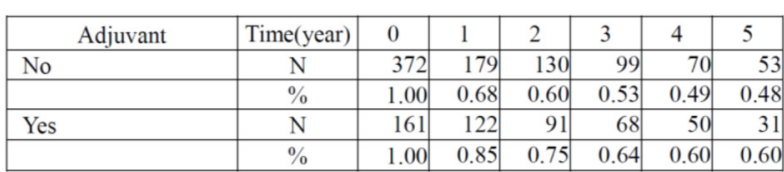

Adjuvant chemotherapy

$\neg$ No

$\neg$ Yes

Figure 1. Kaplan-Meier estimates of overall survival (A), cancer-specific survival (B), and disease-free survival (C) 


\section{Overall survival}

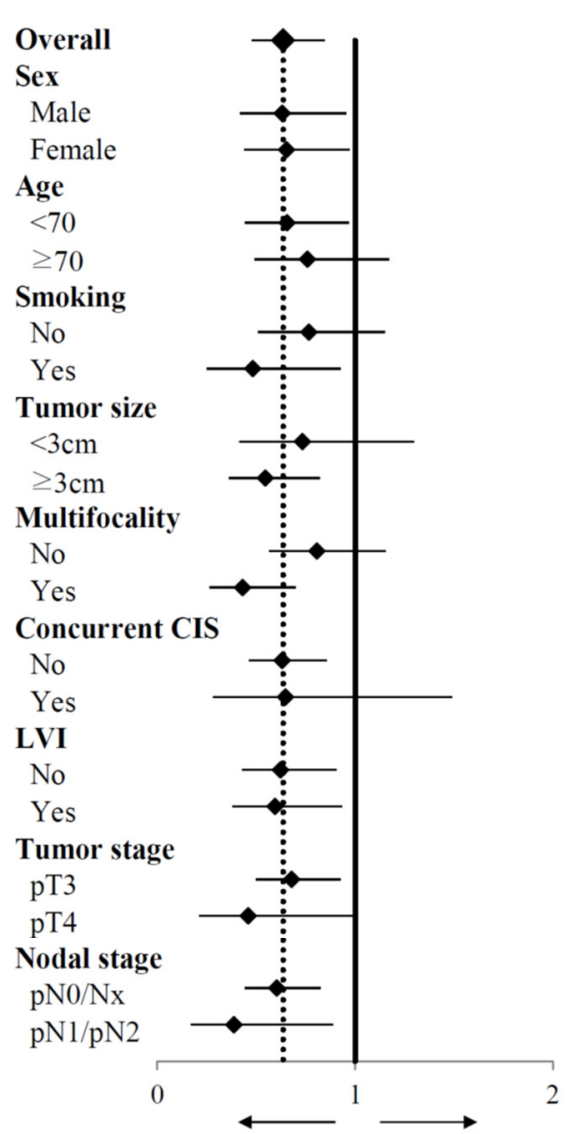

HR $(95 \% \mathrm{CI})$ $0.636(0.478-0.847)$

$0.631(0.418-0.953)$ $0.654(0.439-0.973)$

$0.656(0.444-0.969)$ $0.759(0.492-1.172)$

$0.767(0.511-1.150)$ $0.483(0.252-0.926)$

$0.734(0.415-1.299)$ $0.546(0.364-0.820)$

$0.807(0.564-1.154)$ $0.431(0.267-0.698)$

$0.631(0.466-0.856)$ $0.648(0.282-1.489)$

$0.623(0.429-0.904)$ $0.595(0.379-0.935)$

$0.680(0.499-0.926)$ $0.460(0.214-0.989)$

$0.604(0.442-0.825)$ $0.387(0.169-0.888)$

\section{Cancer-specific survival}

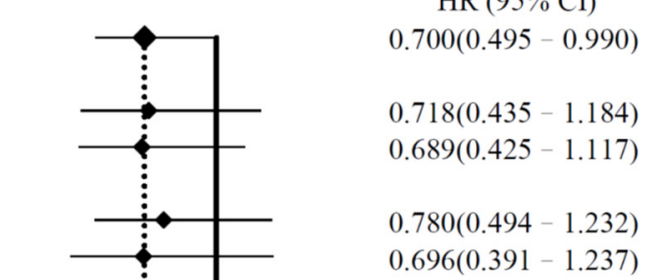

$0.696(0.391-1.237)$

$0.828(0.499-1.372)$ $0.587(0.293-1.179)$

$0.551(0.248-1.225)$ $0.665(0.426-1.038)$

$0.857(0.550-1.335)$ $0.499(0.285-0.876)$

$0.745(0.517-1.074)$ $0.435(0.144-1.316)$

$0.738(0.462-1.177)$ $0.612(0.365-1.027)$

$0.797(0.547-1.162)$ $0.381(0.147-0.988)$

$0.681(0.464-0.999)$ $0.337(0.138-0.827)$

\section{Disease-free survival}

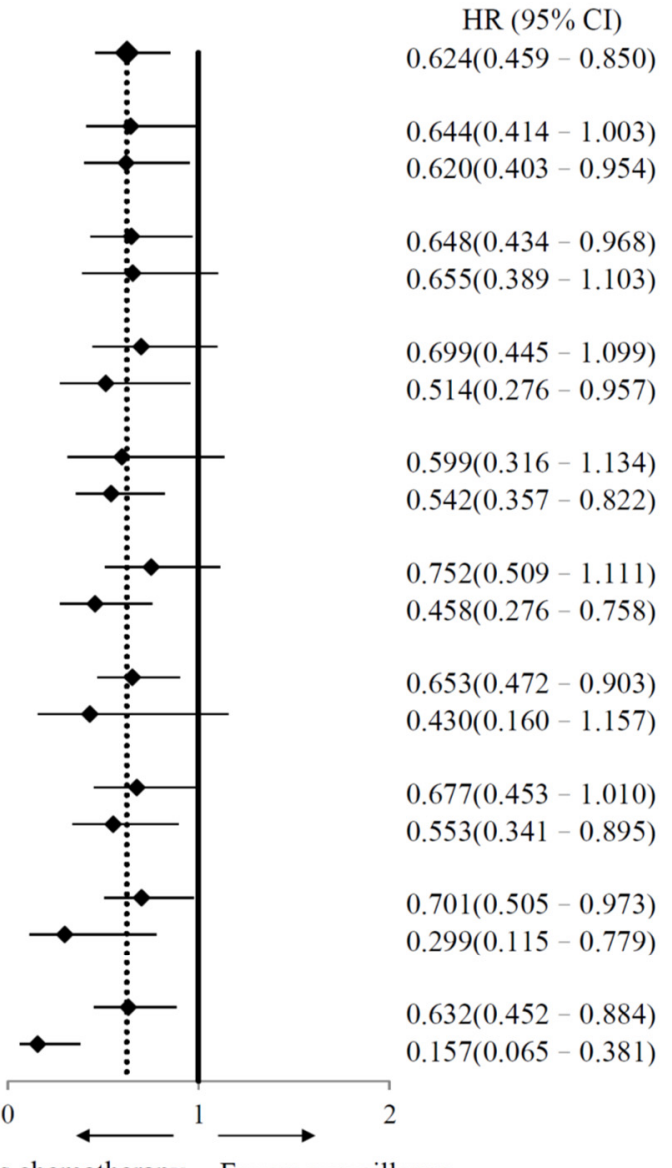

Favors chemotherapy Favors surveillance

Favors chemotherapy Favors surveillance

Figure 2. Subgroup analysis of overall survival, cancer-specific survival, and disease-free survival according to baseline characteristics. HR-hazard ratio; CI-confidence interval; CIS—carcinoma in situ; LVI-lymphovascular invasion. 


\section{Discussion}

The standard procedure for treating UTUC is RNU regardless of the tumor location and excellent oncologic outcomes and lower recurrence rates in T1 and T2 stage disease have been reported $[2,4,5]$. In patients with advanced UTUC, the disease has a high systemic recurrence rate and poor prognosis despite radical surgery $[2,4,5]$. Therefore, adjuvant chemotherapy is considered a reasonable strategy to improve oncologic outcomes and is suggested in the current guidelines [2,11]. Nevertheless, there is currently a lack of data concerning the survival benefit of adjuvant chemotherapy in patients with advanced UTUC, and the data remain controversial and conflicting because of the rarity of UTUC [9]. To address these limitations, we performed an analysis of patients with UTUC from 14 participating Taiwan hospitals, including almost 584 patients with pT3/T4 and without distant metastasis who received either adjuvant chemotherapy or surveillance, respectively.

Previous studies have supported the survival benefits of adjuvant chemotherapy after surgical intervention. In 2020, the first phase III randomized trial (POUT) revealed the benefit of adjuvant systemic chemotherapy after RNU [12]. In 2017, Nakagawa et al. reported that adjuvant chemotherapy improved CSS in a cohort of 109 patients with advanced-stage UTUC after RUN [13]. In 2019, Huang et al. also demonstrated the therapeutic benefit of adjuvant chemotherapy in stage II-IV local UTUC [14].

In the POUT trial [12], the patients were recorded from 71 National Health Service (NHS) hospitals in the UK. Although the race distribution of patients was not mentioned in the POUT study, the underrepresentation of Asian patients in the POUT trial was possible. To the best of our acknowledge, the incidence and presentation of UTUC in the Asian population were different from those in the Western population. We believed our study made up for the lack of evidence and evaluated the effect of adjuvant chemotherapy for East Asian UTUCs patients.

Real-world patients may have poorer performance status and compliance and may include higher proportions of elderly patients. These patients may be excluded from RCT but should not be neglected in our clinical practice. We thought our real-world data were essential and could reflect clinical experience, especially in Asian groups. Our study included patients with high comorbidity (ASA3 and ECOG $\geq 2$ ). We performed subgroup analysis and found adjuvant chemotherapy significantly improved OS, CSS, and DFS in this patient population (Figures S1 and S2). High comorbidities in UTUC may result in competing mortality. We excluded patients who died due to non-cancer before tumor recurrence to minimize competing bias. The results showed that adjuvant chemotherapy was significantly associated with better CSS and DFS (Figure S3). In addition, we also had large case numbers and a long-term follow-up period to strengthen our results. In contrast to our results, Necchi et al. reported that adjuvant chemotherapy was not associated with any survival benefit in high-risk ( $\geq$ pT2 and/or pN1-3) UTUC patients compared to observation following RNU in a retrospective study of 1544 patients [15]. Vassilakopoulou et al. observed no benefits of postoperative chemotherapy in patients with high-risk UTUC [16]. These conflicting results between our study and other studies may have been because both non-cisplatin-based and cisplatin-based adjuvant chemotherapy were included in the study by Necchi et al., and there were unknown regimens in some patients $(115 / 312$, $35.8 \%$ ) [15]. However, most patients in the current study underwent platinum-based adjuvant chemotherapy, including cisplatin and carboplatin. Chemotherapy regimens may have contributed to the differences in results between these studies.

The other possible explanation is the population with metastatic disease in these studies. In our retrospective study, only advanced patients were included. Nevertheless, several other studies enrolled patients with distant metastasis $[15,16]$. Patients with metastatic disease who underwent salvage adjuvant chemotherapy had equivocal benefits in terms of survival outcomes [17]. The benefit may be biased because younger and healthier patients tend to receive chemotherapy, and it may be that the apparent survival time was prolonged. In our opinion, the negative results of these studies were influenced by enrolling patients 
with metastatic disease that shortened the average survival time and contributed to the lack of survival improvement in these studies.

Platinum-based chemotherapy was selected for adjuvant chemotherapy in most patients included in the current study. Moreover, the patients who underwent adjuvant chemotherapy were younger and had a better renal function before and after surgery than those who did not. Patients were treated with a cisplatin-based regimen if the renal function was adequate to tolerate adjuvant chemotherapy. Some patients who had declining renal function and/or decreased tolerability for adjuvant chemotherapy following RUN underwent adjuvant chemotherapy with a carboplatin-based regimen. In a previous study, carboplatin was a useful alternative solution when cisplatin was contraindicated and not at the expense of efficacy [18]. Chang et al. also supported the efficacy of adjuvant chemotherapy with carboplatin in patients with advanced UTUC [14]. In contrast, Leow et al. compared non-cisplatin-based or cisplatin-based adjuvant chemotherapy after RUN and found no survival benefit for non-cisplatin-based regimens [19].

Although the different adjuvant chemotherapy regimens were not a significant prognostic factor in our study, platinum-based chemotherapy for patients with advanced UTUC still had a better treatment effect on survival outcomes than non-platinum-based chemotherapy. Furthermore, the carboplatin-based regimen had relatively better CSS and DFS than the cisplatin-based regimen. This finding contrasts with the results of other studies. In the POUT trial, cisplatin-gemcitabine chemotherapy showed a superior tumor response rate than carboplatin-gemcitabine adjuvant chemotherapy [12]. However, patients with impaired renal function still have survival benefits from the gemcitabine-carboplatin regimen. We cannot be sure why we could not obtain the same result seen in some clinical trials. However, we believe that the following are several possibilities. First, our patients may not be as healthy as the participants in these trials. Good performance status is always an entry criterion for most trials. Second, cisplatin treatment, which follows a strict protocol in prospective trials, is better than clinical practice in reducing mortality. In our opinion, nephrotoxicity of cisplatin may be associated with more complications, such as acute kidney injury, electrolyte imbalance, or erythropoietin deficiency, than carboplatin, which influences the survival outcome in patients with advanced UTUC in real-world practice [20]. The effectiveness of adjuvant chemotherapy with carboplatin-based regimens is still controversial in UTUC. However, our study may provide evidence to support the therapeutic role of carboplatin-based adjuvant chemotherapy in patients with contraindications to cisplatin-based regimens because of impaired renal function.

In our study, the patients who underwent adjuvant chemotherapy had more aggressive pathological features with advanced lymphadenopathy $(>\mathrm{pN} 0)$. At the time of data collection, lymph node dissection (LND) was not a part of standard care because there were little data to support this procedure strongly [2,21]. Nodal dissection has a controversial therapeutic role, and the procedure is performed if nodal metastasis is defined in the clinical image. Dominguez-Escrig et al., Don et al., and Zhai et al. reported that LND had a survival benefit in patients with high stage $(\geq \mathrm{pT} 2)$ UTUC and reduced the risk of local recurrence [22-24]. Guo et al. demonstrated that LND during RUN provides more accurate pathological staging and prediction of prognosis [25]. In our opinion, LND could help physicians identify candidates for adjuvant chemotherapy in patients with advanced UTUC, and our results also reinforced the benefit of adjuvant chemotherapy for pT3/T4N+ patients.

To our knowledge, LVI is a key and significant step in tumor spreading and metastasis and has been regarded as a prognostic factor for UTUC after RNU [2,26-28]. In our study, univariate analysis of survival outcomes revealed that LVI status was associated with worse OS, CSS, and DFS ( $p<0.005)$. However, LVI status became a non-significant predictive factor when other variables were included in the multivariate analysis. This result is similar to that of Chen et al., who reported that LVI was not associated with survival outcomes of UTUC with the pT3 stage [29]. In our opinion, T3 or T4 disease means that tumor cells invade peripheral soft tissue and show a more predominant influence than LVI status on 
survival outcome. The impact of LVI status is minimal in patients with T3/T4 stage and no suitable prognostic factor for survival outcome.

The present study had several limitations. First, this is a retrospective observational study with unavoidable shortcomings, such as inconsistent data collection during the study period, and selection. Evolution of medical care, technology, chemotherapy protocols, and differences in the surgeon experience may also be a source of bias, although the clinical outcomes were not significantly associated with the year of UTUC diagnosis (Figure S4). Second, although the was no significant difference in the patient's general status, more healthy patients were in the adjuvant chemotherapy group. The healthier patient group may contribute to a better clinical outcome and weaken the conclusion of our study. Third, we only perform lymph node dissection in patients with lymph-node involvement identified on preoperative imaging or during surgery. The actually node-positive UTUC disease cannot be completely studied. Last, the patients who received platinum-based chemotherapy were all included regardless of cisplatin or carboplatin in our study, and the cut-off value of the renal function to carboplatin-based regimen might be different in each hospital. A meta-analysis revealed superior tumor response rates in patients with advanced UC treated with cisplatin than those treated with carboplatin [30]. Therefore, the efficacy of adjuvant chemotherapy following RUN may be underestimated. Finally, although our retrospective UTUC cohort was quite large, further prospective, randomized, and multi-institutional studies may be necessary to confirm our results.

\section{Conclusions}

We demonstrated a significant improvement in OS, CSS, and DFS with adjuvant chemotherapy after RNU for patients with advanced UTUC in this large observational study. These results support the use of adjuvant chemotherapy and may assist physicians in patient consultation and clinical decision making.

Supplementary Materials: The following supporting information can be downloaded at: https: //www.mdpi.com/article/10.3390/jpm12020226/s1, Figure S1: Subgroup survival analysis of ASA = 3; Figure S2: Subgroup survival analysis of ECOG > 2; Figure S3: Survival analysis in our cohort excluding non-cancer mortality patients, Figure S4: Survival analysis according to year of UTUC diagnosis, Table S1: Competing risk analyses for cancer-specific and disease-free survivals in advanced UTUC patients.

Author Contributions: Conceptualization, C.-Y.L. and W.-M.L.; methodology, C.-Y.L. and W.-M.L.; data curation, H.-Y.W., T.-Y.T., H.-C.W., W.-C.C., C.-H.C., C.-Y.H., C.-W.L., C.-C.Y., C.-Y.T., W.-C.W., Y.-H.J., Y.-K.L., T.Y.H., A.W.C., B.-J.C., H.-C.H., I.-H.A.C., Y.-T.C., W.-Y.L., C.-C.W., Y.-C.T., H.-Y.L. and W.-M.L.; writing-original draft preparation, C.-Y.L. and H.-Y.L.; writing—review and editing, W.-M.L.; funding acquisition, W.-M.L. All authors have read and agreed to the published version of the manuscript.

Funding: This research was funded by Kaohsiung Medical University Hospital, Taiwan (KMUH1088M53, KMUH109-9R60, KMUH-DK(C)-110006), Kaohsiung Medical University Research Center, Taiwan (KMU-TC109B05), and Ministry of Science and Technology, Taiwan (MOST109-2314-B-037110-MY3, MOST110-2321-B-037-002).

Institutional Review Board Statement: The study was conducted according to the guidelines of the Declaration of Helsinki and approved by the Institutional Review Board of Kaohsiung Medical University Hospital (protocol code KMUHIRB-E(I)-20190107, approved on 8 April 2019).

Informed Consent Statement: IRB approved to waive the need for formal informed consent because this study involved minimal risk. The review of subjects' medical records was for limited information. The information was not sensitive in nature, and the data were derived from clinically indicated procedures.

Data Availability Statement: All data generated or analyzed during this study are included in this published article. 
Acknowledgments: We thank Yu-Tsai Li for statistical assistance and all members of the Taiwan Upper Tract Urothelial Carcinoma Collaboration Group: Allen W. Chiu, Bing-Juin Chiang, ChaoHsiang Chang, Chao-Yuan Huang, Cheng-Huang Shen, Cheng-Kuang Yang, Cheng-Ling Lee, ChenHsun Ho, Che-Wei Chang, Chia-Chang Wu, Chieh-Chun Liao, Chien-HuiOu, Chih-Chen Hsu, ChihChin Yu, Chih-Hung Lin, Chih-Ming Lu, Chih-Yin Yeh, Ching-Chia Li, Chi-Ping Huang, Chi-Rei Yang, Chi-Wen Lo, Chuan-Shu Chen, Chung-Hsin Chen, Chung-You Tsai, Chung-Yu Lin, Chun-Hou Liao, Chun-Kai Hsu, Fang-Yu Ku, Hann-Chorng Kuo, Han-Yu Weng, Hao-Han Chang, Hong-Chiang Chang, Hsiao-Jen Chung, Hsin-Chih Yeh, Hsu-Che Huang, Ian-Seng Cheong, I-Hsuan Alan Chen, Jen-Kai Fang, Jen-Shu Tseng, Jen-Tai Lin, Jian-Hua Hong, Jih-Sheng Chen, Jungle Chi-Hsiang Wu, Kai-Jie Yu, Keng-Kok Tan, Kuan-Hsun Huang, Kun-Lin Hsieh, Lian-Ching Yu, Lun-Hsiang Yuan, Hao-LunLuo, Marcelo Chen, Min-Hsin Yang, Pai-Yu Cheng, Po-Hung Lin, Richard Chen-Yu Wu, See-Tong Pang, Shin-Hong Chen, Shin-Mei Wong, Shiu-Dong Chung, Shi-Wei Huang, Shuo-Meng Wang, Shu-Yu Wu, Steven Kuan-Hua Huang, Ta-Yao Tai, Thomas Y. Hsueh, Ting-En Tai, Victor ChiaHsiang Lin, Wei-Chieh Chen, Wei-Ming Li, Wei-Yu Lin, Wen-Hsin Tseng, Wen-Jeng Wu, Wun-Rong Lin, Yao-Chou Tsai, Yen-Chuan Ou, Yeong-Chin Jou, Yeong-Shiau Pu, Yi-Chia Lin, Yi-Hsuan Wu, Yi-Huei Chang, Yi-sheng Lin, Yi-Sheng Tai, Yu-Khun Lee, Yuan-Hong Jiang, Yu-Che Hsieh, Yu-Chi Chen, Yu-Ching Wen, Yung-Tai Chen, and Zhe-Rui Yang.

Conflicts of Interest: The authors declare no conflict of interest.

\section{References}

1. Soria, F.; Shariat, S.F.; Lerner, S.P.; Fritsche, H.-M.; Rink, M.; Kassouf, W.; Spiess, P.E.; Lotan, Y.; Ye, D.; Fernandez, I.M.; et al. Epidemiology, diagnosis, preoperative evaluation and prognostic assessment of upper-tract urothelial carcinoma (UTUC). World J. Urol. 2017, 35, 379-387. [CrossRef] [PubMed]

2. Rouprêt, M.; Babjuk, M.; Burger, M.; Capoun, O.; Cohen, D.; Compérat, E.M.; Cowan, N.C.; Dominguez-Escrig, J.L.; Gontero, P.; Mostafid, A.H.; et al. European Association of Urology Guidelines on Upper Urinary Tract Urothelial Carcinoma: 2020 Update. Eur. Urol. 2020, 79, 62-79. [CrossRef] [PubMed]

3. Chou, Y.H.; Huang, C.H. Unusual clinical presentation of upper urothelial carcinoma in Taiwan. Cancer 1999, 85, 1342-1344. [CrossRef]

4. Li, C.-C.; Chang, T.-H.; Wu, W.-J.; Ke, H.-L.; Huang, S.-P.; Tsai, P.-C.; Chang, S.-J.; Shen, J.-T.; Chou, Y.-H.; Huang, C.-H. Significant Predictive Factors for Prognosis of Primary Upper Urinary Tract Cancer after Radical Nephroureterectomy in Taiwanese Patients. Eur. Urol. 2008, 54, 1127-1135. [CrossRef] [PubMed]

5. $\quad$ Li, W.-M.; Shen, J.-T.; Li, C.-C.; Ke, H.-L.; Wei, Y.-C.; Wu, W.J.; Chou, Y.-H.; Huang, C.-H. Oncologic Outcomes Following Three Different Approaches to the Distal Ureter and Bladder Cuff in Nephroureterectomy for Primary Upper Urinary Tract Urothelial Carcinoma. Eur. Urol. 2010, 57, 963-969. [CrossRef] [PubMed]

6. Colin, P.; Koenig, P.; Ouzzane, A.; Berthon, N.; Villers, A.; Biserte, J.; Rouprêt, M. Environmental factors involved in carcinogenesis of urothelial cell carcinomas of the upper urinary tract. Br. J. Urol. 2009, 104, 1436-1440. [CrossRef]

7. Wang, Y.-H.; Yeh, S.-D.; Shen, K.-H.; Shen, C.-H.; Juang, G.-D.; Hsu, L.-I.; Chiou, H.-Y.; Chen, C.-J. A significantly joint effect between arsenic and occupational exposures and risk genotypes/diplotypes of CYP2E1, GSTO1 and GSTO2 on risk of urothelial carcinoma. Toxicol. Appl. Pharmacol. 2009, 241, 111-118. [CrossRef] [PubMed]

8. Margulis, V.; Shariat, S.F.; Matin, S.F.; Kamat, A.M.; Zigeuner, R.; Kikuchi, E.; Lotan, Y.; Weizer, A.; Raman, J.; Wood, C.G.; et al. Outcomes of radical nephroureterectomy: A series from the Upper Tract Urothelial Carcinoma Collaboration. Cancer 2009, 115, 1224-1233. [CrossRef]

9. Abouassaly, R.; Alibhai, S.; Shah, N.; Timilshina, N.; Fleshner, N.; Finelli, A. Troubling Outcomes From Population-level Analysis of Surgery for Upper Tract Urothelial Carcinoma. Urology 2010, 76, 895-901. [CrossRef]

10. Witjes, J.A.; Bruins, H.M.; Cathomas, R.; Compérat, E.M.; Cowan, N.C.; Gakis, G.; Hernández, V.; Espinós, E.L.; Lorch, A.; Neuzillet, Y.; et al. European Association of Urology Guidelines on Muscle-invasive and Metastatic Bladder Cancer: Summary of the 2020 Guidelines. Eur. Urol. 2021, 79, 82-104. [CrossRef]

11. Flaig, T.W.; Spiess, P.E.; Agarwal, N.; Bangs, R.; Boorjian, S.A.; Buyyounouski, M.K.; Chang, S.; Downs, T.M.; Efstathiou, J.A.; Friedlander, T.; et al. Bladder Cancer, Version 3.2020, NCCN Clinical Practice Guidelines in Oncology. J. Natl. Compr. Cancer Netw. 2020, 18, 329-354. [CrossRef]

12. Birtle, A.; Johnson, M.; Chester, J.; Jones, R.; Dolling, D.; Bryan, R.; Harris, C.; Winterbottom, A.; Blacker, A.; Catto, J.W.F.; et al. Adjuvant chemotherapy in upper tract urothelial carcinoma (the POUT trial): A phase 3, open-label, randomised controlled trial. Lancet 2020, 395, 1268-1277. [CrossRef]

13. Nakagawa, T.; Komemushi, Y.; Kawai, T.; Otsuka, M.; Miyakawa, J.; Uemura, Y.; Kanatani, A.; Taguchi, S.; Naito, A.; Suzuki, M.; et al. Efficacy of post-nephroureterectomy cisplatin-based adjuvant chemotherapy for locally advanced upper tract urothelial carcinoma: A multi-institutional retrospective study. World J. Urol. 2017, 35, 1569-1575. [CrossRef] 
14. Chang, Y.; Hsiao, P.; Chen, G.; Lin, C.; Chang, C.; Wu, H.; Huang, C.; Yang, C.; Yeh, S. Outcomes of stage II-IV upper-tract urothelial carcinoma and adjuvant chemotherapy for locally advanced cancer. Oncol. Lett. 2018, 17, 1341-1348. [CrossRef] [PubMed]

15. Necchi, A.; Vullo, S.L.; Mariani, L.; Moschini, M.; Hendricksen, K.; Rink, M.; Sosnowski, R.; Dobruch, J.; Raman, J.D.; Wood, C.G.; et al. Adjuvant chemotherapy after radical nephroureterectomy does not improve survival in patients with upper tract urothelial carcinoma: A joint study by the European Association of Urology-Young Academic Urologists and the Upper Tract Urothelial Carcinoma Col. Br. J. Urol. 2018, 121, 252-259. [CrossRef] [PubMed]

16. Vassilakopoulou, M.; Rouge, T.D.L.M.; Colin, P.; Ouzzane, A.; Khayat, D.; Dimopoulos, M.; Papadimitriou, C.A.; Bamias, A.; Pignot, G.; Nouhaud, F.X.; et al. Outcomes after adjuvant chemotherapy in the treatment of high-risk urothelial carcinoma of the upper urinary tract (UUT-UC). Cancer 2011, 117, 5500-5508. [CrossRef] [PubMed]

17. Ikeda, M.; Matsumoto, K.; Hirayama, T.; Koguchi, D.; Murakami, Y.; Matsuda, D.; Okuno, N.; Utsunomiya, T.; Taoka, Y.; Irie, A.; et al. Oncologic Outcomes of Salvage Chemotherapy in Patients with Recurrent or Metastatic Lesions after Radical Nephroureterectomy: A Multi-Institutional Retrospective Study. Chemotherapy 2020, 65, 134-140. [CrossRef] [PubMed]

18. Ho, G.Y.; Woodward, N.; Coward, J.I. Cisplatin versus carboplatin: Comparative review of therapeutic management in solid malignancies. Crit. Rev. Oncol. 2016, 102, 37-46. [CrossRef]

19. Leow, J.J.; Martin-Doyle, W.; Fay, A.P.; Choueiri, T.K.; Chang, S.L.; Bellmunt, J. A Systematic Review and Meta-analysis of Adjuvant and Neoadjuvant Chemotherapy for Upper Tract Urothelial Carcinoma. Eur. Urol. 2014, 66, 529-541. [CrossRef]

20. Miller, R.P.; Tadagavadi, R.K.; Ramesh, G.; Reeves, W.B. Mechanisms of Cisplatin Nephrotoxicity. Toxins 2010, 2, 2490-2518. [CrossRef]

21. Seisen, T.; Shariat, S.F.; Cussenot, O.; Peyronnet, B.; Renard-Penna, R.; Colin, P.; Rouprêt, M. Contemporary role of lymph node dissection at the time of radical nephroureterectomy for upper tract urothelial carcinoma. World J. Urol. 2017, 35, 535-548. [CrossRef] [PubMed]

22. Dominguez-Escrig, J.L.; Peyronnet, B.; Seisen, T.; Bruins, H.M.; Yuan, Y.; Babjuk, M.; Böhle, A.; Burger, M.; Compérat, E.M.; Gontero, P.; et al. Potential Benefit of Lymph Node Dissection During Radical Nephroureterectomy for Upper Tract Urothelial Carcinoma: A Systematic Review by the European Association of Urology Guidelines Panel on Non-muscle-invasive Bladder Cancer. Eur. Urol. Focus 2019, 5, 224-241. [CrossRef] [PubMed]

23. Dong, F.; Xu, T.; Wang, X.; Shen, Y.; Zhang, X.; Chen, S.; Zhong, S.; Zhang, M.; Ding, Q. Lymph node dissection could bring survival benefits to patients diagnosed with clinically node-negative upper urinary tract urothelial cancer: A population-based, propensity score-matched study. Int. J. Clin. Oncol. 2018, 24, 296-305. [CrossRef] [PubMed]

24. Zhai, T.-S.; Jin, L.; Zhou, Z.; Liu, X.; Liu, H.; Chen, W.; Lu, J.-Y.; Yao, X.-D.; Feng, L.-M.; Ye, L. Effect of lymph node dissection on stage-specific survival in patients with upper urinary tract urothelial carcinoma treated with nephroureterectomy. BMC Cancer 2019, 19, 1207. [CrossRef]

25. Guo, R.; Zhu, Y.; Xiong, G.; Li, X.; Zhang, K.; Zhou, L. Role of lymph node dissection in the management of upper tract urothelial carcinomas: A meta-analysis. BMC Urol. 2018, 18, 24. [CrossRef]

26. Novara, G.; Matsumoto, K.; Kassouf, W.; Walton, T.J.; Fritsche, H.-M.; Bastian, P.J.; Martínez-Salamanca, J.I.; Seitz, C.; Lemberger, R.J.; Burger, M.; et al. Prognostic Role of Lymphovascular Invasion in Patients with Urothelial Carcinoma of the Upper Urinary Tract: An International Validation Study. Eur. Urol. 2010, 57, 1064-1071. [CrossRef]

27. Liu, W.; Zhou, Z.; Dong, D.; Sun, L.; Zhang, G. Prognostic Value of Lymphovascular Invasion in Node-Negative Upper Urinary Tract Urothelial Carcinoma Patients Undergoing Radical Nephroureterectomy. Yonsei Med. J. 2019, 60, 174-181. [CrossRef]

28. Fan, E.W.; Li, C.-C.; Wu, W.J.; Huang, C.-N.; Li, W.-M.; Ke, H.-L.; Yeh, H.-C.; Wu, T.-F.; Liang, P.-I.; Ma, L.-J.; et al. FGF7 Over Expression is an Independent Prognosticator in Patients with Urothelial Carcinoma of the Upper Urinary Tract and Bladder. J. Urol. 2015, 194, 223-229. [CrossRef]

29. Chen, C.-S.; Lin, C.-Y.; Wang, C.-L.; Wang, S.-S.; Li, J.-R.; Yang, C.-K.; Cheng, C.-L.; Chiu, K.-Y.; Yang, S.-F. Association between lymphovascular invasion and oncological outcome in node-negative upper tract urothelial carcinoma with different stage. Urol. Oncol. Semin. Orig. Investig. 2021, 39, 132.e13-132.e26. [CrossRef]

30. Galsky, M.D.; Chen, G.J.; Oh, W.; Bellmunt, J.; Roth, B.J.; Petrioli, R.; Dogliotti, L.; Dreicer, R.; Sonpavde, G. Comparative effectiveness of cisplatin-based and carboplatin-based chemotherapy for treatment of advanced urothelial carcinoma. Ann. Oncol. 2012, 23, 406-410. [CrossRef] 